\title{
Knowledge across networks: how to build a global neuroscience collaboration
}

\author{
Lauren E. Wool ${ }^{\mathrm{a}, *}$ and The International Brain Laboratory \\ anstitute of Neurology, University College London, Cruciform Building, Gower Street, London \\ WC1E 6BT \\ ${ }^{*}$ Corresponding author: lauren.e.wool@gmail.com
}

\section{Highlights}

- The International Brain Laboratory (IBL) is a flexible, distributed, and predominantly virtual network of neuroscientists studying decision-making and the mouse brain.

- IBL researchers (postdocs, students, staff) are a diverse group of scientists essential for sustaining and refining this collaborative network.

- Intersecting knowledge domains (formal, contextual, experimental, theoretical) are critical for successful collaborative neuroscience research.

- Flat organizational hierarchies encourage researcher advocacy, to refine and reform research culture and scientific practice.

\begin{abstract}
The International Brain Laboratory (IBL) is a collaboration of $\sim 20$ laboratories dedicated to developing a standardized mouse decision-making behavior, coordinating measurements of neural activity across the mouse brain, and utilizing theoretical approaches to formalize the neural computations that support decision-making. In contrast to traditional neuroscientific practice, in which individual laboratories each probe different behaviors and record from a few select brain areas, IBL aims to deliver a standardized, high-density approach to behavioral and neural assays. This approach relies on a highly distributed, collaborative network of $\sim 50$ researchers-postdocs, graduate students, and scientific staff-who coordinate the intellectual, administrative, and sociological aspects of the project. In this article, we examine this network, extract some lessons learned, and consider how IBL may represent a template for other team-based approaches in neuroscience, and beyond.
\end{abstract}

\section{Introduction}

The International Brain Laboratory (IBL) was founded with the goal of understanding how the brain operates to drive decision-making behavior [1]. Decision-making behavior demands information about sensory stimuli, reward expectation, and prior experience, processed by millions of neurons within local circuits and across brain regions. Fully understanding these processes is of a scale and complexity far exceeding what can be tackled by any single laboratory. As such, the IBL's approach is to implement a distributed research architecture across a global network of laboratories to assay how decision-making behavior is encoded in 
the mouse brain. This architecture combines a high-throughput behavioral task with high-density neural recordings, standardized anatomical mapping, and collaborative code repositories for analysis and modeling -all alongside a commitment to publicly deposit our data, tools, and resources for everyone to use.

The collaboration is currently distributed over 22 laboratories across six countries, with each location hosting several members who implement IBL tools and resources within the existing laboratory infrastructure. Our network has unified the efforts of nearly 80 neuroscientists to develop an experimental ecosystem generating high-throughput behavioral data in mice [2], a pipeline for acquiring large-scale, high-density neural recordings during behavior, and a public-facing digital architecture to host these data [3].

IBL was organized because 'team science' consistently demonstrates high impact [4], innovation [5], and productivity [6]. As a distributed team model, we have coalesced resources around a unifying scientific question while also accommodating members' autonomy within traditional institutions, where full interdisciplinary collaboration can be cumbersome to implement $[7,8]$. The result is a low-hierarchy organization of scientists that remain independent in skills, research interests, and physical location, who contribute individual expertise toward a shared methodology and develop a common vocabulary in order to communicate across large-scale projects $[9,10]$.

Specifically, transmuting team-science philosophy into tangible outputs is due to the efforts of IBL's scientific researchers. Postdocs, graduate students, and scientific staff have built a complex, grassroots team-science apparatus [11] that lets us connect existing labs and traditional institutions, distribute tasks and resources, and produce resources for the greater scientific community. As our collaboration enters its fourth year, we take this opportunity to examine its organizational structure, extract the lessons learned, and understand how it may serve as a model for other team-based approaches in neuroscience (Box 1).

\section{'Researchers': a unified identity}

Alongside the 22 faculty members who lead their lab's participation in the collaboration, $\sim 50$ additional IBL members are responsible for the collaboration's day-to-day scientific activities. Many studies differentiate science workers by career stage [12], but we found common terms like 'early career researchers' or 'trainees' failed to capture the breadth and variety of experience in our cohort. This group includes postdocs, students, technicians, staff, software developers, and industry contractors, each with different scientific vocabularies, lab experiences, and educational backgrounds. Despite these differences, many of us participate at the front lines of many different scientific activities such as writing software, building hardware, recording neurons, handling animals, leading team meetings, analyzing data, or writing papers - to name only a few. As such, we collectively identify as 'researchers'. Applying a single unified identity across traditional academic boundaries has greatly influenced the knowledge 
exchange across the collaboration, and has expanded our definition of what constitutes scientific work.

\section{Local and global crossover}

$\mathrm{IBL}$ researchers are a mix of members who contribute full- or part-time to IBL initiatives. A small core of full-time IBL staff members develop and maintain key platforms for IBL research, like data architecture or technical infrastructure. Of the $\sim 30$ postdocs and graduate students, roughly half work full-time on IBL-specific scientific projects, while the rest share their time between IBL projects and research in their local labs. Our research technicians, software developers, and industry contractors also divide their efforts between both local and IBL research activities.

Since researchers participate in a local research environment while also participating in IBL's, the boundary between local and IBL-wide activities is permeable. This enables research cultures to intersect and encourages exchange with other systems neuroscience initiatives beyond IBL. For instance, while we acquired expertise from our local labs to inform IBL's standard experimental protocols, we liaise with other neuroscientists who want to adopt the resulting tools [2] for their own projects. Crossover efforts between projects within and beyond $\mathrm{IBL}$ have been an opportunity for us to collectively develop and refine approaches across systems neuroscience more broadly.

\section{Making a virtual space}

Compared to collaborations that develop their practices within a single physical institution [13], IBL developed across multiple, distributed locations and relies on a virtual group [14]. The IBL virtual environment resembles other modern 'postwork' environments, in which digital sharing bends the spatial and temporal boundaries of the workplace [15]. The collaboration itself is physically distributed across 16 institutions and nine time zones, but real-time online connectivity erases these boundaries and instead supports a centralized space where IBL members, irrespective of physical location, regularly convene to maintain knowledge and share resources. Just as in a traditional laboratory, members collectively manage a mix of interdependent empirical, technical, and administrative tasks, all united by a set of scientific goals.

Our virtual environment is scaffolded by a combination of Slack (real-time messaging), Google GSuite (documentation), Zoom (video conferencing), Github (code repository), and Datajoint and Alyx (experimental databases). While these tools are an indispensable part of IBL's online environment, we still periodically utilize one or two traditional in-person meetings each year to build cohesion and community beyond digital spaces. Our annual meeting convenes all members to discuss scientific milestones and team-wide policies, while an annual researcher summit affords an independent space to navigate the successes and challenges specific to postdocs, students, and staff. 


\section{Flat hierarchies facilitate access}

IBL's virtual environment has grown to accommodate a diversity of scientific activity, and is supported by a flexible, 'flattened' hierarchy (Figure 1) that emphasizes horizontal relationships over vertical management [16]. This structure is directly inspired by the ATLAS collaboration at CERN, which has a voting assembly to ratify decisions, an executive body managing operations, and a flexible network of specialized subgroups [17]. Similarly, small teams of IBL members collaborate on projects in Working Groups (WGs), which are defined around particular specializations and milestones (Table 1) and coordinated jointly by a chair and associate chair (typically a PI and researcher, respectively). All WG chairs sit on the Executive Board to propagate decisions across WGs, facilitate operational and financial support, and prepare proposals for voting by the General Assembly, which represents all PIs. In parallel, associate chairs convene on their own committee to share decisions, which are then conveyed to the entire researcher community so it may weigh in on proposals before a formal vote. The interests of PIs and researchers intersect via staff liaisons who sit on both the Executive Board and the Associate Chairs Committee, as well as an elected researcher representative, who sits on the Executive Board and is a voting member of the General Assembly.

IBL's governance structure is complemented by our use of "sociocracy", a form of dynamic consent-based decision-making that encourages members to iteratively create actions that are "good enough for now, safe enough to try" [18]. Proposals are developed by PIs and researchers alike, from all corners of the collaboration, and are announced in team-wide spaces to facilitate input not only from vested stakeholders or formal representatives, but by all IBL members directly. This model of rapid iteration, stakeholder crossover, and direct access has made it easy for researchers to interact and support each other on various tasks and policies. It has also diminished the hierarchy between Pls and researchers, facilitating access to senior members for other, broader goals—scientific engagement, academic supervision, and professional support.

\section{Knowledge occupies people and places}

Across our virtual environment, information in the IBL is open-all records are accessible by any member, irrespective of position or length of membership. While this open access has facilitated a culture of transparency [19], the total amount of information generated within the collaboration presents an enormous practical obstacle: as of April 2020, there were 34,478 public Slack messages; 363 recorded Zoom meetings; 187 presentation slide decks, 233 spreadsheets, 1,618 text documents, and 7,603 PDFs in GSuite; 6,148 Github commits; and over 500,000 experimental files in Datajoint. The IBL ecosystem, like other biological and social systems, is a network [20], but successfully distributing this much information across it is a daunting proposition.

Two essential features coordinate this information to better serve our organizational decision-making, learning, and memory [21]. The first is our constellation of Working Groups 
that maintain and distribute local, specialized knowledge to other groups across the network. Since each IBL researcher typically participates in three or four Working Groups, there is a high degree of information exchange, which juxtaposes related initiatives and complementary expertise (Figure 2). A second, more emergent property is the subgroup of IBL researchers who have become experts, liaisons, and interpreters of knowledge across the network [22]. These members each manage a domain of explicit records (e.g., written protocols) and tacit information (e.g., colloquialisms, decision histories) that are quickly and informally disseminated to address real-time needs and problems. A remarkable nimbleness is afforded by this system of rapid responders deployed across our web of Working Groups. However, this kind of internalized knowledge can be vulnerable to drop-out when people leave the collaboration, and can be complex to archive [23]. An ongoing challenge for our collaboration is how to archive both our explicit and tacit processes held in both people and places. This is not only to document our own history but as part of a roadmap for future science teams, whose dynamics are still not fully understood [24].

\section{Strengthening formal knowledge with context}

In traditional laboratories, a mix of both academic trainees and staff seems to foster scientific progress [25], which can be attributed to the exchange of trainees' 'formal' knowledge of scientific phenomena (e.g., theories, papers, or grants) with staff's 'contextual' knowledge of how those phenomena manifest in the lab (e.g., materials, instruments, or techniques) [26]. Knowledge is similarly allocated within IBL in that trainees focus on generating papers and staff focus on maintaining infrastructure, but in a flat hierarchy, this allocation is flexible. IBL trainees regularly engage with instrumentation or back-end software development, while staff often participate in experimental design, data visualization, or analysis. This exchange enables a more comprehensive understanding of data generated in our laboratory spaces. Take, for example, a recent staff-postdoc collaboration that synthesized animal husbandry techniques and behavioral analysis: ad libitum access to $2 \%$ citric acid water works as well as traditional water restriction for task-performing mice [27].

Across science, increasingly capricious funding and a hypercompetitive trainee pipeline have arguably led to a culture of conservative, short-term scientific returns [28]. However, diversifying the laboratory with researchers with complementary skills and career trajectories could help guide a shift toward more creative, sustainable scientific growth. Many scientific questions being addressed are also too ambitious to be tackled by a single trainee, and are increasingly relying on large, longitudinal research teams concentrated in physical institutes like Janelia Research Campus [29] and the Allen Institute [13]. While physical proximity between collaborating researchers has its advantages, IBL's distributed model allows team composition to be adjusted more flexibly and nimbly, drawing together talents from around the world. Indeed, our own platform papers [2,3] highlight the sheer diversity of researchers needed to complete these large-scale projects. Expanding researcher roles beyond trainees should be a central goal if our field wishes to stabilize large-scale collaborative science in traditional academia [30]; it is 
promising to consider the new types of researchers we can attract if we don't need to bring them into a single lab, but rather can bring the lab directly to them.

\section{Experiments need theories, and vice versa}

If formal-contextual exchange is one axis of IBL's knowledge sharing, another is the interchange between theory and experiment, an implicit partnership underlying all scientific outputs [31]. On one hand, theory can be iterated to produce models that satisfy constraints imposed by empirical data; on the other, experimentation is disciplined by hypotheses generated from models. Addressing this directly, a critical IBL mandate [11] is to ensure that theory and experiment converge at the ground level, and perpetually throughout our scientific process.

Although most IBL member labs still largely identify as either 'experimental' or 'theoretical', IBL's virtual network means that experimental and theoretical researchers are constantly interacting. An experimentalist/theorist 'buddy system' offers a low-stakes, one-on-one partnership where we can ask each other general or introductory questions, while further crossover is encouraged through an online computational neuroscience course led by theorists for experimentalists, and a sabbatical initiative, where a researcher can embed within a partner lab to learn firsthand new concepts. Sharing theory-experiment spaces on multiple levels has allowed researchers to synthesize biological and mathematical vocabularies to develop a common language that bridges this traditional divide. Maintaining this crossover should continue to help us derive more complete neuroscientific answers, but also formulate more relevant questions [32].

\section{Committing to transparent histories}

Scientific findings result from the culmination of many actions that generate theories, data, analyses, and interpretations. However, a complete history of these actions is rarely ever recorded - this could be due to long research timeframes [33], short institutional memories [34], space constraints in a 'single, flat PDF' [13], or a reward system that favors paper counts over good laboratory habits [35-37]. It is possible that incompletely recording and reviewing actions in the lab could contribute to irreproducibility in systems neuroscience [38-40] and science in general [41-43].

How, then, to produce a more transparent and documented scientific process? Two elements of IBL's approach seem to hold promise. First, the connections between researchers and Working Groups (Figure 2) afford a great deal of redundancy in our information transfer, and enable us to examine findings through a diversity of experimental, technical, and theoretical lenses. Multiple researchers interact with bits of data or theories as they emerge, and while this process generates a rich complex of individual contributions, it invites many members into collective responsibility for any single scientific result [44]. Second, we have built publication and data policies that mandate public sharing of data, methods, code, and software, as well as open-access publications via Plan S [45]. This ensures that our findings are shared with communities of both scientists and the public for further analysis and inspection, as a "trust technology" [46]. 
Maintaining complete and transparent histories for our platform projects has been particularly demanding given their size and scale. The number of contributions that any single researcher makes to an IBL project far exceeds what is typically enshrined in print. IBL's behavioral platform paper [2], for instance, has 26 researchers named as coauthors and a contribution statement of over 1300 words (consider that Nature's current formatting guidelines recommend "several sentences"). This is strong evidence that scientific collaborations are outgrowing the limitations of traditional authorship, which will require expanding our taxonomies of credit [47] and creatively managing the diverse efforts that go into large-scale projects. An ongoing IBL effort is to develop a comprehensive credit-assignment system that reflects a spirit of collaboration while also providing meaningful signifiers of individuals' work that translate beyond the collaboration.

\section{Advocating for change}

Developing a successful credit-assignment system is just one of many ongoing evolutions in $\mathrm{IBL}$-most of them spearheaded by researchers. Despite the already-immense job pressures [48], we spend considerable time assessing IBL practices and proposing overhauls through a combination of annual reports and real-time proposals. We have led actions on project registration and organizational record-keeping; policies on environmental responsibility, safety climate, diversity and inclusion, and professional development; and even petitions to scientific journals to expand guidelines to accommodate IBL's authorship scheme. Cultivating advocacy alongside science research may be one of the enduring touchstones of IBL's collaborative model, providing next-generation scientists with agency to tackle the issues in academia and reinvent a beleaguered system. IBL's model has already provided fertile ground for testing many new scientific practices. Perhaps the next iteration will have even less resemblance to traditional science, further pushing toward a fully collaborative, reproducible, and open endeavor-and making room for an even greater diversity of scientists who come after us.

\section{Data and code availability}

Network analysis was completed using NetworkX [49] and Plotly [50] Python libraries. Data and code are available on Figshare (https://figshare.com/projects/IBL_Network_Analysis/84239).

\section{Acknowledgments}

Many thanks to all of the IBL researchers for rich discussions over the past three years that helped shape the structure and message of this article. Particular thanks goes to Z.C. Ashwood, H.M. Bayer, G.A. Chapuis, M. Häusser, A. Pouget, M.M. Schartner, A. Urai, and M.R. Whiteway for constructive comments on the manuscript. L.E.W. is a member of IBL, which is funded by the Simons Foundation and the Wellcome Trust. L.E.W. is also supported by a Marie Skłodowska-Curie Actions individual fellowship (no. 795846). 


\section{Declarations of interest}

The authors declare no competing interests.

\section{Figures}

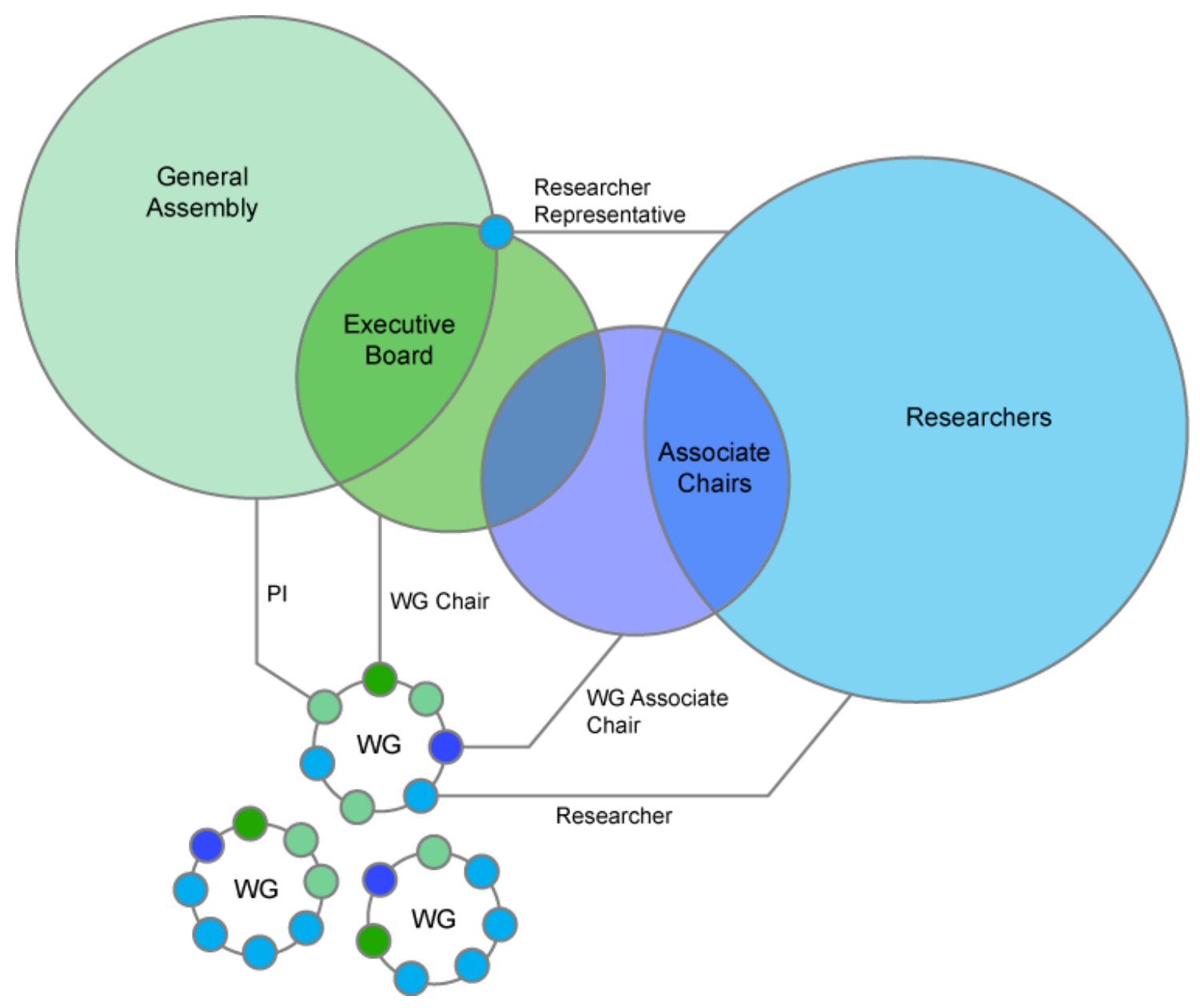

Figure 1. The IBL 'flat hierarchy' model. On a day to day level, small teams of IBL members collaborate on projects in Working Groups (WGs), which are defined around particular specializations and milestones (Table 1) and coordinated jointly by a chair and associate chair (typically a PI and researcher, respectively). Decisions and proposals generated in WGs take two parallel paths through the organization. For PI-focused stakeholder groups (green), WG chairs convene on the Executive Board to share decisions across WGs, facilitate operational and financial support, and formalize proposals for voting by the General Assembly, which represents all PIs. For researcher-focused stakeholder groups (blue), associate chairs convene to share decisions across WGs, which are then conveyed to all researchers for a chance to influence proposals directly. $\mathrm{Pl}$ and researcher interests interconnect via staff liaisons who sit on both the Executive Board and the Associate Chairs Committee, as well as by an elected researcher representative, who sits on the Executive Board and is a voting member of the General Assembly. Lines between groups illustrate how stakeholders are represented in WGs; for clarity, only a few lines for one WG are shown. 


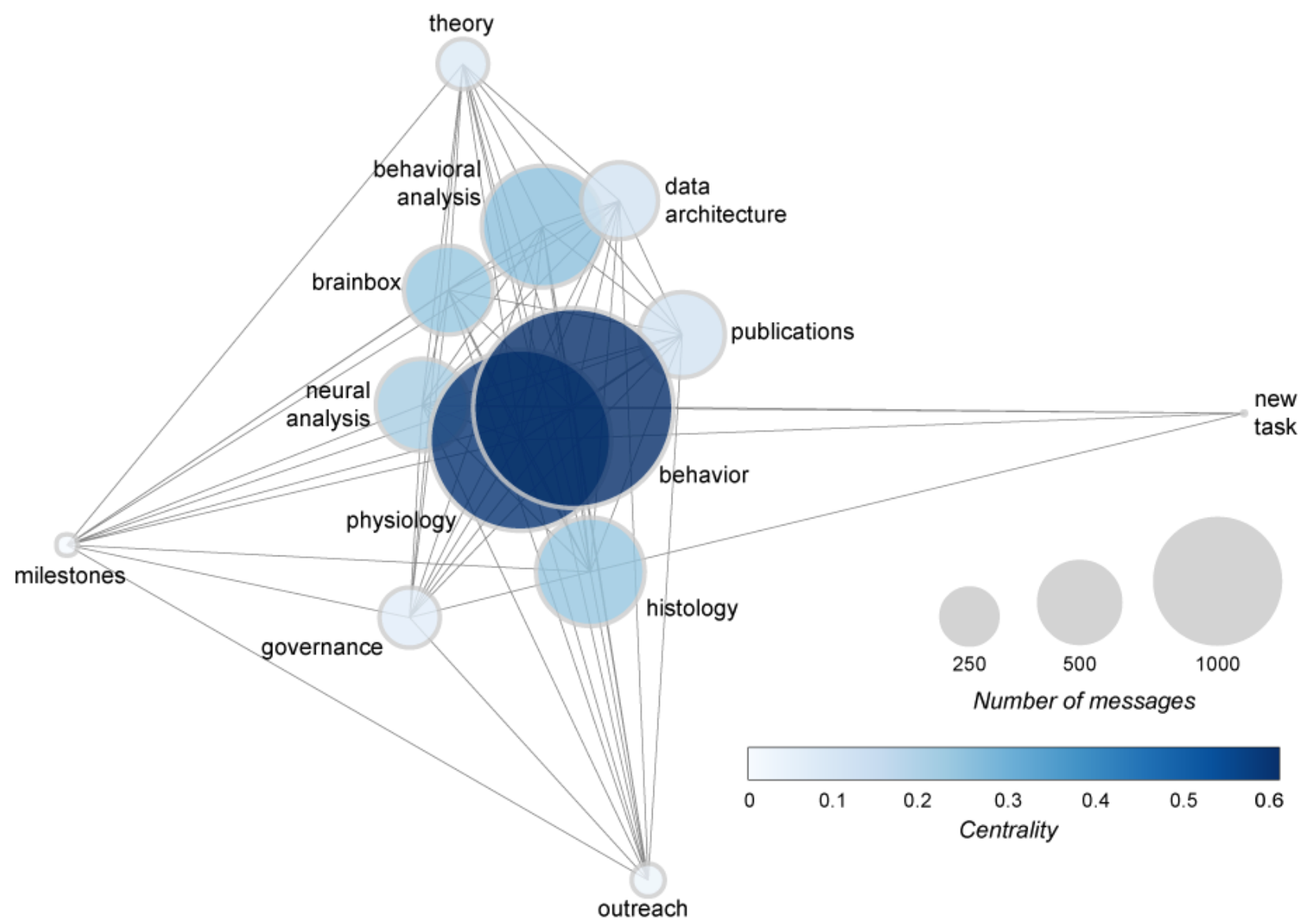

Figure 2. IBL's network of Working Groups, as viewed through 9,226 Slack messages sent by 41 researchers to 13 Working Group channels (August 2016-April 2020). Each node represents a working group, and each edge between two nodes represents a researcher who messaged both groups. Since some researchers message more than others, each researchers' message count was normalized as a proportion. Researchers who sent no messages to these channels were omitted from analysis. Node position and edge length were determined using edge weight, which was computed as the product of message proportion between the edge's two nodes. Node shade represents the eigenvector centrality as determined from the weights of all connected edges. Node size reflects the absolute message count to that group. Connections between Working Groups are stronger (shorter edges, higher centrality) when they share more researchers and/or messages, and weaker (longer edges, lower centrality) when they share fewer researchers and/or messages. Data for this analysis is available in Google Colab (see 'Data and code availability'). 
Box 1. Ten thoughts on building a large-scale scientific collaboration.

1. Develop common language to connect your team. Overlapping Working Groups help build shared technical vocabularies, and using a single 'researchers' label connects many different IBL scientists with different experience, education, and career trajectories.

2. Keep a flexible boundary between internal and external projects. Giving ourselves time to engage in both IBL and local lab projects has broadened the uptake of our resources and involved many outside colleagues as collaborators.

3. Create and maintain a centralized environment to exchange knowledge and build community. For virtual teams like ours, this has required several different online tools and information formats.

4. Keep organizational hierarchy 'flat' so different stakeholders can interact and influence policy. We broadly distribute decisions through parallel channels and share policy centrally so that each member can contribute directly or through a representative.

5. Cultivate information in different spaces across your organization, and anticipate that some team members will become key facilitators of this information. Working Groups are essential bodies of specialized knowledge in $\mathrm{IBL}$, and 'expert' members connect others to this knowledge.

6. Acknowledge that organizational knowledge occupies different forms, locations, and people, which makes archiving complex and challenging. Despite this, commit to documenting your process. IBL members are constantly refining strategies for documenting decisions and project progress.

7. Anticipate that developing large-scale resources will require a diversity of efforts and a rich crossover of knowledge domains. Make space for those in one domain to contribute to another. Crossovers between formal vs. contextual knowledge, as well as experimental vs. theoretical knowledge, are constant features of IBL projects.

8. Adhere to open-source principles and make public sharing a foundational expectation. This will diminish organizational boundaries, encourage transparency, and expand your impact. IBL's data and publication policies mandate public sharing, to broadly inform neuroscience and influence research culture in general.

9. Prepare to longitudinally track contributions to projects as they grow, and utilize a taxonomy that can accommodate new, unique and diverse contributions. The IBL behavioral platform paper has been documenting contributions for nearly 12 months, and is actively developing a comprehensive credit-assignment policy for its platform papers.

10. Make the changes needed to push your team forward. Alongside science work, IBL researchers routinely advocate to improve research culture and respond to issues facing scientific practice both within and beyond the collaboration. 
Table 1. Working Groups and objectives as of April 30, 2020.

\begin{tabular}{|c|c|c|}
\hline Name & Objective & Created \\
\hline Governance & $\begin{array}{l}\text { Manage, review and help implement policies for } \\
\text { the collaboration's function }\end{array}$ & 25 August 2016 \\
\hline $\begin{array}{l}\text { Neural } \\
\text { Analysis }\end{array}$ & Analyze neural data & 7 September 2016 \\
\hline Publication & $\begin{array}{l}\text { Develop policies and guidelines pertaining to } \\
\text { scientific communication, authorship, and credit }\end{array}$ & 14 September 2016 \\
\hline Physiology & Develop tools and protocols for neural recordings & 25 September 2016 \\
\hline Behavior & $\begin{array}{l}\text { Develop and deploy the behavioral task in } \\
\text { member labs }\end{array}$ & 10 January 2018 \\
\hline $\begin{array}{l}\text { Behavioral } \\
\text { Analysis }\end{array}$ & Curate, visualize, and analyze behavioral data & 16 July 2018 \\
\hline $\begin{array}{l}\text { Data } \\
\text { Architecture }\end{array}$ & $\begin{array}{l}\text { Design and implement the platform for hosting } \\
\text { and sharing IBL data }\end{array}$ & 20 March 2019 \\
\hline Histology & $\begin{array}{l}\text { Design and implement processing pipeline for } \\
\text { analyzing post-experiment brain tissue }\end{array}$ & 20 March 2019 \\
\hline Theory & $\begin{array}{l}\text { Develop canonical models for animal behavior } \\
\text { and neural activity }\end{array}$ & 20 March 2019 \\
\hline Brainbox & $\begin{array}{l}\text { Develop and curate a Python toolbox for neural } \\
\text { and behavioral analysis }\end{array}$ & 17 June 2019 \\
\hline Outreach & $\begin{array}{l}\text { Coordinate activities for scientific communication } \\
\text { and public engagement }\end{array}$ & 24 September 2019 \\
\hline New Task & Discuss and develop future behavioral tasks & 21 November 2019 \\
\hline $\begin{array}{l}\text { Scientific } \\
\text { Milestones }\end{array}$ & $\begin{array}{l}\text { Discuss and develop future projects under the } \\
\text { IBL banner }\end{array}$ & 17 December 2019 \\
\hline
\end{tabular}




\section{References}

1. The International Brain Laboratory: An International Laboratory for Systems and Computational Neuroscience. Neuron 2017, 96:1213-1218.

2. The International Brain Laboratory, Aguillon-Rodriguez V, Angelaki DE, Bayer HM, Bonacchi N, Carandini M, Cazettes F, Chapuis GA, Churchland AK, Dan Y, et al.: A standardized and reproducible method to measure decision-making in mice. bioRxiv 2020, doi:10.1101/2020.01.17.909838.

3. The International Brain Laboratory, Bonacchi N, Chapuis G, Churchland A, Harris KD, Rossant C, Sasaki M, Shen S, Steinmetz NA, Walker EY, et al.: Data architecture and visualization for a large-scale neuroscience collaboration. bioRxiv 2019, doi:10.1101/827873.

4. Wuchty S, Jones BF, Uzzi B: The Increasing Dominance of Teams in Production of Knowledge. Science 2007, 316:1036-1039.

5. Uzzi B, Mukherjee S, Stringer M, Jones B: Atypical Combinations and Scientific Impact. Science 2013, 342:468-472.

6. Hall KL, Stokols D, Stipelman BA, Vogel AL, Feng A, Masimore B, Morgan G, Moser RP, Marcus SE, Berrigan D: Assessing the Value of Team Science: A Study Comparing Center- and Investigator-Initiated Grants. Am J Prev Med 2012, 42:157-163.

7. Kaplan S, Milde J, Cowan RS, Kleinman D, Mccray P, Mody C, Nelson A, Piore M: Interdisciplinarity in Practice: A Case of a Nanotechnology Research Center. 2012,

8. Vogel AL, Stipelman BA, Hall KL, Nebeling L, Stokols D, Spruijt-Metz D: Pioneering the Transdisciplinary Team Science Approach: Lessons Learned from National Cancer Institute Grantees. J Transl Med Epidemiol 2014, 2.

9. Jeffrey P: Smoothing the Waters: Observations on the Process of Cross-Disciplinary Research Collaboration. Soc Stud Sci 2003, 33:539-562.

10. Mody CCM: Instrumental Community: Probe Microscopy and the Path to Nanotechnology. Mit Press; 2011.

11. Mainen ZF, Häusser M, Pouget A: A better way to crack the brain. Nature 2016, 539:159-161.

12. Laudel G, Gläser J: From apprentice to colleague: The metamorphosis of Early Career Researchers. High Educ 2008, 55:387-406.

13. Koch C, Jones A: Big Science, Team Science, and Open Science for Neuroscience. Neuron 2016, 92:612-616.

14. Lipnack J, Stamps J: Virtual Teams: Reaching Across Space, Time and Organizations with Technology by Jessica Lipnack. John Wiley \& Sons; 1997.

15. Richardson L: Sharing as a postwork style: digital work and the co-working office. Camb J Reg Econ Soc 2017, 10:297-310.

16. Prunty R: Flattening Organizations. Glob Encycl Public Adm Public Policy Gov 2016,

17. Tuertscher $P$, Garud R, Kumaraswamy A: Justification and Interlaced Knowledge at ATLAS, CERN. Organ Sci 2014, 25:1579-1608.

The authors comprehensively review the organizational structure of the ATLAS collaboration at CERN, a distributed model of team science that strongly influenced IBL's organization. The article introduces the term interlaced knowledge-the concept where pockets of shared knowledge intereave within and across subsystem communities.

18. Buck JA, Villines S: We the people: consenting to a deeper democracy : a guide to sociocratic principles and methods. Sociocracy.info; 2007. 
19. Schnackenberg AK, Tomlinson EC: Organizational Transparency: A New Perspective on Managing Trust in Organization-Stakeholder Relationships. J Manag 2016, 42:1784-1810.

20. Ahn Y-Y, Bagrow JP, Lehmann S: Link communities reveal multiscale complexity in networks. Nature 2010, 466:761-764.

21. Cyert RM, March JG: A Behavioral Theory of the Firm. Prentice Hall; 1963.

22. Huber GP: Organizational Learning: The Contributing Processes and the Literatures. Organ Sci 1991, 2:88-115.

This review article formalizes the term organizational learning as the phenomenon of team-wide growth, development, or forgetting, and delineates a number of processes and subprocesses by which this occurs in interpersonal networks. While organizational science historically pertained to firms or business, Huber's concepts are apt for today's science teams and our understanding of how knowledge grows and transforms within collaborations.

23. Cross R, Baird L: Technology Is Not Enough: Improving Performance by Building Organizational Memory. Sloan Manag Rev Camb 2000, 41:69-78.

24. Hall KL, Vogel AL, Huang GC, Serrano KJ, Rice EL, Tsakraklides SP, Fiore SM: The science of team science: A review of the empirical evidence and research gaps on collaboration in science. Am Psychol 2018, 73:532-548.

This work reviews the Science of Team Science (SciTS), a field with the goal of empirically addressing questions from funding agencies, administrators, and scientists regarding the value of team science. This review summarizes the empirical findings from the SciTS literature and explores policies and practices for implementing and sustaining successful science teams.

25. Conti A, Liu CC: Bringing the lab back in: Personnel composition and scientific output at the MIT Department of Biology. Res Policy 2015, 44:1633-1644.

26. Barley SR, Bechky BA: In the Backrooms of Science: The Work of Technicians in Science Labs. Work Occup 1994, 21:85-126.

27. Urai AE, Aguillon-Rodriguez V, Laranjeira IC, Cazettes F, Laboratory TIB, Mainen ZF, Churchland AK: Citric Acid Water as an Alternative to Water Restriction for High-Yield Mouse Behavior. bioRxiv 2020, doi:10.1101/2020.03.02.973016.

28. Alberts $B$, Kirschner MW, Tilghman S, Varmus H: Rescuing US biomedical research from its systemic flaws. Proc Natl Acad Sci 2014, 111:5773-5777.

29. Rubin GM, O'Shea EK: Looking back and looking forward at Janelia. eLife 2019, 8:e44826.

30. National Research Council: Enhancing the Effectiveness of Team Science. 2015.

31. Guest $O$, Martin AE: How computational modeling can force theory building in psychological science. PsyArXiv 2020, doi:10.31234/osf.io/rybh9.

While specifically aimed at the psychological sciences, the authors make a universal argument that computational models are critical for principled interpretation of empirical data. Their "path model" for maximizing the relationship across theory, specification, and data is accessible and approachable for those wishing to better integrate their workflows.

32. Smaldino P: Better methods can't make up for mediocre theory. Nature 2019, 575:9.

33. Powell K: Does it take too long to publish research? Nat News 2016, 530:148.

34. Pollitt C: Institutional Amnesia: A Paradox of the "Information Age"? Prometheus 2000, 18:5-16.

35. Brischoux F, Angelier F: Academia's never-ending selection for productivity. Scientometrics 2015, 103:333-336. 
36. Horton R: Offline: What is medicine's 5 sigma? The Lancet 2015, 385:1380.

37. Smaldino PE, McElreath R: The natural selection of bad science. $R$ Soc Open Sci 2016, 3:160384.

38. Tuttle AH, Philip VM, Chesler EJ, Mogil JS: Comparing phenotypic variation between inbred and outbred mice. Nat Methods 2018, 15:994-996.

39. Chesler EJ, Wilson SG, Lariviere WR, Rodriguez-Zas SL, Mogil JS: Influences of laboratory environment on behavior. Nat Neurosci 2002, 5:1101-1102.

40. Crabbe JC, Wahlsten D, Dudek BC: Genetics of Mouse Behavior: Interactions with Laboratory Environment. Science 1999, 284:1670-1672.

41. Baker M: 1,500 scientists lift the lid on reproducibility. Nat News 2016, 533:452.

42. McElreath R, Smaldino PE: Replication, Communication, and the Population Dynamics of Scientific Discovery. PLOS ONE 2015, 10:e0136088.

43. Pashler H, Harris CR: Is the Replicability Crisis Overblown? Three Arguments Examined: Perspect Psychol Sci 2012, doi:10.1177/1745691612463401.

44. Simone D: Copyright and Collective Authorship: Locating the Authors of Collaborative Work. Cambridge University Press; 2019.

In this comprehensive analysis of copyright law, Simone considers how collective authorship informs a variety of highly collaborative works-from Wikipedia to filmmaking, Aboriginal art, and scientific collaborations-and proposes an inclusive and contextual redefinition that stays true to the legal concept of authorship but better aligns with creative reality.

45. Schiltz M: Science Without Publication Paywalls: cOAlition S for the Realisation of Full and Immediate Open Access. PLOS Med 2018, 15:e1002663.

46. Grand A, Wilkinson C, Bultitude K, Winfield AFT: Open Science: A New "Trust Technology"? Sci Commun 2012, 34:679-689.

47. Holcombe A: Farewell authors, hello contributors. Nature 2019, 571:147-147.

48. Freeman R, Weinstein E, Marincola E, Rosenbaum J, Solomon F: Competition and Careers in Biosciences. Science 2001, 294:2293-2294.

49. Hasberg AA, Schult DA, Swart PJ: Exploring network structure, dynamics, and function using NetworkX. In Proceedings of the 7th Python in Science Conference (SciPy2008). Edited by Varoquaux G, Vaught T, Millman J. 2008:11-15.

50. Plotly Technologies Inc: Collaborative data science. 2015. 